Revista

\title{
Multi-Ensayos
}

Vol. 6, núm. 11

ISSN: 2412-3285

https://multiensayos.unan.edu.ni

DOI: https://doi.org/10.5377/multiensayos.v6i11.9291

\section{Una buena práctica de empresa, es clave para el desarrollo profesional de estudiantes de Energía Renovable}

\section{Good business practice is key to the professional development of Renewable Energy students}

\author{
Juan Alberto Betanco Maradiaga
}

\section{RESUMEN}

Recibido: 24 de octubre de 2019. Aceptado: 14 de enero de 2020

El ingeniero de hoy debe saber hacer las cosas, manejar muy bien los aspectos de su especialidad, tener capacidad de aprender lo que necesite ante los problemas que surjan y nunca olvidar el aspecto humano. La formación universitaria del ingeniero requiere énfasis en que las prácticas de formación que realiza el estudiante en las empresas sean de calidad. Las reflexiones en el presente ensayo giran en torno a experiencias de estudiantes de IV año de ingeniería en Energias Renovables en su práctica de especialización. Se han considerado aspectos relacionados a la importancia del ingeniero para la sociedad, los aspectos curriculares que sustentan el desarrollo de las prácticas de especialización, la interacción de la academia, la empresa y el estado como sistema de fortalecimiento educativo mientras se forma el profesional, se culmina con evidencias de algunas vivencias en actividades especializadas que ejecutaron los jóvenes practicantes incidiendo positivamente en su desarrollo profesional.

Palabras claves: Ingeniería; prácticas de especialización; energías renovables; relación universidadestado-empresa.

\section{ABSTRACT}

Today's engineers must know how to do things and handle the skills of his specialty very well. Engineers can learn what they need to solve problems that arise without forgetting the human aspect. The engineer's university training requires an emphasis on the practicum quality that trainees perform in the companies. This essay reflects on the experiences of 4th-year Renewable Energy Engineering students who completed their specialization practicum. Aspects related to the importance of engineering for society, the curricular elements that underpin the development of specialization practicum, the interaction of academia companies, and the state as a strengthening system for the formation of the professional have been considered. It culminates with evidence of some experiences in specialized activities that were carried out by the young practitioners, which impacted positively on their professional development.

Keywords: Engineering; specialization practices; renewable energies; university-state-company relationship.

1 Docente UNAN-Managua/FAREM-Estelí. Correo electrónico: juan.betanco@yahoo.com. (c) 2020 Revista Multi-Ensayos. 


\section{INTRODUCCIÓN}

Siempre ha sido una preocupación de los docentes del ámbito universitario, la formación de un ingeniero que responda al entorno social, que responda a las necesidades del contexto en el que vive y se desenvuelve, que pueda hacer las cosas, resolverlas y enfrentar, que sepa aplicar conocimientos para enfrentar la realidad, que use la ciencia para hacer y usar tecnología que transforme los recursos disponibles para satisfacer necesidades.

Al alcanzar el séptimo semestre los estudiantes de ingeniería renovables han obtenido conocimientos sobre evaluaciones de recursos energéticos, circuitos eléctricos, electrónica, termometría y termodinámica, energía solar fotovoltaica. Ha llegado el momento de integrarse al proceso productivo en diversas empresas del sector energético y asumir el reto de demostrar lo que saben, profundizar conocimiento y desenvolverse en otro entorno que no es el aula de clase.

A pesar de las prácticas de laboratorio, las simulaciones, las resoluciones de ejercicios, las conferencias, los seminarios en ingeniería, el aprendizaje no está completo sino se tiene la vivencia de la realidad que genera nuevas formas de pensamiento y de actuar. Es por eso que en el presente ensayo se argumenta basados en aquel viejo refrán "la práctica hace al maestro" y a afirmar que "La realización de las prácticas en una empresa especializada en energías renovables es condicionante para profundizar el conocimiento en el desarrollo del futuro profesional".

El presente ensayo, tiene el propósito de compartir las experiencias de los estudiantes de IV año de ingeniería en Energías Renovables de la Facultad Regional Multidisciplinaria Estelí (FAREM Estelí) de la UNAN Mangua, en la asignatura de prácticas de especialización. Se abordan la fundamentación teórica sobre las prácticas de profesionalización, el modelo de relación universidad con el entorno productivo y la experiencia del aprender haciendo para generar conocimiento más profundo.

\section{DESARROLLO}

\section{El aprendizaje en la ingeniería}

Los países necesitan profesionales bien formados que participen en los procesos de investigación, innovación y desarrollo al más alto nivel. También necesitan ciudadanos capaces de manejarse y participar en un mundo en el que las decisiones de base científica forman parte cotidiana de la vida, elemento este que requiere una adecuada respuesta desde las instituciones educativas (Rizo, 2007).

La actividad profesional de los ingenieros se sitúa en el corazón de los procesos económicos de las sociedades, pues esta profesión ha sido tradicionalmente la encargada de aplicar los conocimientos científicos al desarrollo, operación y mantenimiento de sistemas y a su optimización en términos de calidad y coste (Recuero López, 2002). 
La enseñanza de la ingeniería desde su surgimiento ha estado condicionada por diferentes cambios que la han hecho evolucionar y a la vez enriquecerse. La enseñanza de la ingeniería reclama necesidades y exigencias para lograr que el proceso de formación responda a las exigencias del contexto, aspecto este que demanda una organización del proceso docente educativo centrado en el estudiante, desarrollado de manera interactiva y colaborativa y que le permita adquirir un aprendizaje para toda la vida (Capote León, Rizo Rabelo, \& Bravo López, 2016).

El modelo educativo (UNAN Managua, 2011, pág. 7),

Consciente de su responsabilidad histórica, y de acuerdo con su Misión, asume la responsabilidad de formar profesionales mediante la gestión de un Modelo Educativo centrado en las personas, que contribuye con el desarrollo integral de los estudiantes. Este Modelo articula las acciones de los diferentes actores partícipes en el quehacer educativo de la Universidad y, además, orienta la formación de profesionales con una concepción científica y humanista, capaces de interpretar los fenómenos sociales y naturales con un sentido crítico, reflexivo y propositivo.

El desarrollo de este tipo de profesionales en el siglo XXI requiere asumir desafíos complejos sobretodo en América Latina que sigue atravesando convulsiones políticas en un mundo polarizado, desigualdades en lo científico-tecnológico, globalización económica y una inmensa brecha en la equidad y justicia social. Esta complejidad obliga a pensar en profesionales con una formación holística capaces de generar cambios sociales; por lo tanto, el aprendizaje debe estar basado en los saberes estratégicos aprender a aprender, aprender hacer, aprender a vivir juntos, aprender a ser, aprender a emprender, aprender a crear. Según (Castellanos Álvarez, Lara Díaz, \& Lapido Rodríguez, 2004), la educación holística concibe la formación de ingenieros en términos de integración e interrelaciones, como un sistema vivo, dinámico, como una comunidad de aprendizaje que posibilite un método para aprender y enseñar. Su propósito fundamental es el desarrollo integral del estudiante de ingeniería como ser humano y como solucionador de problemas en su contexto social, con un alto sentido de compromiso y responsabilidad.

\section{Las prácticas de especialización en ingeniería renovables}

Las prácticas de profesionalización del ingeniero permiten consolidar y fortalecer conocimientos adquiridos en la universidad. Es en las prácticas donde el estudiante se da cuenta para que le sirve lo que aprendió, en que aspecto tiene que profundizar la lectura, lo que le falta por aprender. Esa experiencia la logra en una buena empresa de su área que lo integre a procesos laborales, que le motive a participar con confianza. El modelo de UNAN Managua (UNAN Managua, 2011, pág. 68), sobre las prácticas de profesionalización menciona, "El desarrollo de las prácticas profesionales se conceptualiza como una práctica académica estudiantil, que contribuye a consolidar los conocimientos, habilidades y destrezas adquiridas por los y las estudiantes, así como el desarrollo de actitudes y valores. Son parte sustancial del Plan de Estudios y tienen como objetivo vincular interdisciplinariamente al estudiante en su futuro campo laboral". 
UNAN Managua determina tres tipos de prácticas para todas las carreras de su oferta educativa; familiarización, especialización y profesionalización. En relación a las prácticas de especialización las define (2011), como la fase que permitirá al estudiante definir el área de especialización que más le interesa, ya que según el Perfil Profesional tiene la posibilidad de desempeñarse en varias áreas. Con estas prácticas tendrá la oportunidad de relacionar la teoría con la práctica, lo que, a su vez, garantiza la aplicación del conocimiento científico adquirido en los diferentes campos de su profesión. Esta fase brinda la posibilidad de llevar a cabo proyectos de atención a necesidades específicas en un área de especialización profesional (pág. 69). El modelo no menciona si estas prácticas se deben realizar en las empresas.

El programa de asignatura prácticas de especialización (Betanco M \& Lanuza S, 2016, pág. 4) para el estudiante de ingeniería renovables según el plan de estudio

Se ubica en el VII semestre de la malla curricular, porque el estudiante ya posee una base sólida formada por asignaturas de formación general, de formación básica y formación profesionalizante que le permite desenvolverse en el entorno laboral de la práctica. Tiene como requisito la asignatura de Práctica de Familiarización, se consideran como asignaturas precedentes a Energía Solar Fotovoltaica, Termometría y Termodinámica, Laboratorio de Mediciones Eléctricas y Electrónica. Todas estas asignaturas aportan elementos técnicos, habilidades y destrezas para el efectivo desempeño de la práctica en mención. Esta asignatura se vincula con asignaturas posteriores como Energía Solar Térmica, Hidroenergía y Energía Eólica.

En el aspecto técnico (Betanco M \& Lanuza S, 2016), las Prácticas de Especialización deben permitir a los estudiantes comprender y manejar las diferentes opciones de tecnología, dimensionamiento de sistema y viabilidad de proyectos. Esta práctica tiene el propósito de lograr que los estudiantes se especialicen en una rama de las energías renovables que puede ser en el área de térmica, fotovoltaica, eólica e hídrica mediante el desarrollo de proyectos y estudios de casos.

La ubicación de los estudiantes en las empresas y entidades se basa en la estrategia de vincular universidad, empresa y el estado para mejorar la competitividad (Rodriguez P, 2016, pág. 8), "La vinculación Universidad- Empresa- Estado se ha erigido en las últimas décadas como una estrategia de cambio permanente para mejorar la competitividad de los sectores productivos, contribuir a la solución de problemas de la sociedad y, lo más importante, armonizar sus programas mediante su capacidad de cambio y actualización con las exigencias de los mercados". Esto trae beneficio para todos los involucrados en la triada vinculante "Las instituciones de educación superior y la entidad productiva o de servicios y del Estado, cuando se vinculan se benefician ambas partes y contribuyen de modo esencial al cumplimiento de sus misiones formativas, investigativas y productivas del país" (Rodriguez P, 2016, pág. 9).

Según (Bodas Gonzáles, 2019), las empresas, o más ampliamente las entidades colaboradoras, desempeñan un papel imprescindible para que la formación proporcionada por la universidad se complemente con la adquirida en contextos reales de trabajo de forma que, a través de las prácticas, se ajuste en mayor medida a las necesidades y demandas del mercado laboral. 


\section{Relación Universidad - Estado - Empresa}

La Facultad Regional Multidisciplinaria FAREM Estelí ha aprovechado las buenas relaciones con el estado y las empresas para ubicar a los estudiantes en empresas apropiadas a cada área de conocimiento obteniendo resultados satisfactorios para todos los participantes. En el área de ingenierías renovables se ha establecido vínculos con ENEL central, ente regulador que tiene que ver con las generadoras de energía estatales hidroeléctricas Centroamérica, Carlos Fonseca, la geotérmica Momotombo. Con la empresa privada en el occidente del país se tienen cooperaciones con el tecnológico de la Salle, la geotérmica de San Jacinto Tizate Polarys, Eco energía, ENICLSA y en Estelí con Cubas Eléctrica, ECAMI, TECNOSOL, EMSE renovable, otras empresas como GRID y mujeres solares del grupo FENIX entre otras.

Todas las empresas anteriores tienen una amplia trayectoria profesional con misión y visión definidas y están constituidas por varios departamentos que permiten al estudiante de práctica rotar por diversas áreas técnicas, control y administrativas. La elección de una buena empresa para la realización de la práctica de especialización es el primer paso para garantizar buenos resultados y para comprobar el nivel del ingeniero que se forma en la facultad. Algunas críticas han surgido del hecho de ¿Por qué los estudiantes tienen que viajar hasta el occidente del país para hacer sus prácticas generando más gastos para ellos? Es sencillo, en el occidente del país por la cadena volcánica situada en esa zona y su alta radiación solar es el lugar de mayor aprovechamiento de los recursos energéticos renovables que ha conllevado a consolidar empresas en la zona. En cuanto a los gastos los estudiantes ahorran con anterioridad y se juntan para alquilar hospedajes a menor precio y preparar sus propios alimentos. Esta sinergia entre ellos se ve compensada con mayores aprendizajes y trabajo en equipo, de suma importancia para el ámbito laboral.

\section{Experiencias del aprendizaje en las prácticas de especialización}

Los estudiantes de IV año de energías renovables realizaron 240 horas de prácticas de especialización en empresas energéticas estatales y privadas, integrándose de miércoles a sábado de cada semana. Los practicantes y los responsables en los centros de prácticas consideran que se han logrado avances en el aprendizaje y en el fortalecimiento de conocimientos especializado. He aquí algunas de las experiencias informadas por los estudiantes que contribuyen a ratificar la significancia para el desarrollo de cada uno de ellos mediante el aprender - haciendo.

Keydell Pastrana estudiante de IV año de ingeniería en energías renovable fue seleccionada de entre cuatro compañeros de estudio, por autoridades de ENEL Central para desarrollar su práctica de especialización en la planta hidroeléctrica Carlos Fonseca Amador, ubicada en el Valle "La Rauda" municipio de Ciudad Darío en el Departamento de Matagalpa, a $149.0 \mathrm{~km}$ de la capital Managua y a $67 \mathrm{~km}$ de la ciudad de Estelí. Keydell Pastrana (comunicación personal, 17 agosto 2019) menciona "Durante el proceso de desempeño de la práctica de especialización realice un sinnúmero de actividades que permitieron un desarrollo de destreza y habilidades relacionadas con mi profesión. Estas fueron muy fundamentales en mi crecimiento profesional y por ende en mi desarrollo personal". 
Su primera tarea fue identificar las partes de la hidroeléctrica a nivel de esquema (figura 1 en anexo) y real. Realizó actividades medioambientales establecimiento y manejo de vivero, jornadas de limpiezas en áreas de planta, entrega de plantas forestales a dueños de fincas aledaños a las fuentes de agua. En el área de operaciones hizo labores de manejo del sistema SCADA, que consiste en controlar y monitorear los diferentes elementos de la central hidroeléctrica (figura 2 en anexos), conocer elementos que complementan al sistema de la central hidroeléctrica, lecturas de hoja diaria de control suministro de energía y capacidad de los elementos, encendido de unidades por sistema, elaboración de informes de estados en que se encuentra las maquinarias, embalse, y otros, al hacer cambio de turno. Keydell en su presentación de informe habló con propiedad sobre las actividades mencionadas demostrando que sus prácticas de especialización fueron de mucho provecho.

En el informe de práctica (Castillo, 2019) menciona que Momotombo es el área geotérmica más estudiada de Nicaragua y su primera investigación fue en 1966, en mil 1974 fue la primera perforación de un pozo de exploración, en el campo hay 44 pozos, 27 de estos oscilan en profundidades de entre $328 \mathrm{~m}$ y 1500 $\mathrm{m}$ y 11 de estos tienen profundidades que varían entre $1500 \mathrm{~m}$ y 2000 m, los últimos 6 son pozos de 2000 y 2500 m. El estudiante de IV año de ingeniería en energías renovables Jeffry Alexander Castillo Rugama fue ubicado en ENEL Central en Managua y desde ahí visitaba juntos a ingenieros eléctricos la geotérmica de Momotombo y las diversas plantas hidroeléctricas.

Entre las actividades realizadas figuraron conocer el funcionamiento, instalaciones y los tipos de mantenimiento que se realizan en la planta Momotombo, hizo visita de reconocimiento a diferentes pozos e instalaciones del campo Momotombo como pozos productores, pozos re-inyectores, pozos de monitoreo, planta binaria, sala de monitoreo, cuarto de máquinas de la planta convencional y torres de enfriamiento. Jeffry tuvo la experiencia de analizar una problemática de campo como es la deposición de sílice o dióxido de silicio (SiO2) que se da por la precipitación y los cambios bruscos de temperatura. En el pozo de producción MT-36 se observó gran cantidad de sílice incrustada en el vertedero. Participó en la toma de registro de presión y temperatura del pozo mencionado; con el objetivo de evaluar la viabilidad de una acidificación para recuperar la producción del pozo.

La alianza con otras universidades permitió que Priscila Castro Rodríguez realizará sus prácticas de especialización en el centro de Investigación e Innovación para el Desarrollo Tecnológico de Energías Alternativas (CIDTEA) de la universidad tecnológica La Salle León EI CIDTEA, es un centro dedicado a impulsar la investigación en cuanto a las potencialidades y aplicación de las energías renovables, se enfocan en temas como la producción de Biodiesel a partir de los aceites vegetales de desecho y la producción y aplicación del Biogás.

Entre las actividades realizadas por Priscila destacan elaboración de protocolo de investigación, sistematización del proceso de producción de biocombustible realizado en La ULSA (figura 4 en anexo), asesoría en el proceso de elaboración de trabajos de fin de curso en diferentes áreas temáticas: Física, química, dinámica, modificación de motor diésel para que funcione con biodiesel. 
Mediante la relación con empresas productivas tres estudiantes Wilfredo Ramírez, Rosa Melania Arauz y Seyling Siles Picado se integraron en su práctica de especialización a la reconocida empresa de León, ECOENERGIA. Esta es una empresa dedicada a la Energía Solar Fotovoltaica y Térmica, instalando sistemas con baterías aislados de la red, sistemas conectados a la red, bombeo de agua con energía solar, calentamiento de agua sanitaria y bombeo solar en piscinas.

Se integraron a actividades productivas realizadas por ECOENERGIA como instalaciones de sistemas de bombeo solar, instalaciones de sistema solar fotovoltaico (figura 5 en anexos) montaje de un sistema hibrido, mantenimiento de baterías y bombas sumergible (figura 6 en anexos). Reforzaron conocimientos sobre el dimensionado de sistemas solares. Aprendieron sobre el funcionamiento de máquinas eléctricas y no eléctricas y el manejo de instrumentos de medición que desconocían. En el aspecto actitudinal destacan haber adquirido capacidad de trabajar bajo presión, comunicación asertiva y buenas relaciones laborales.

La empresa ENICALSA de León proporcionó la oportunidad para que cuatro estudiantes de energías renovables, William Vanegas, Douglas Ríos Olivas, Víctor Ruiz y Ángel Darine Rugama se integraran a labores productivas en sus prácticas de especialización. ENICALSA es una empresa cuya misión es, satisfacer las necesidades energéticas básicas con energías alternativas en áreas donde las energías convencionales no lo contemplen.

Los estudiantes practicantes en ENICALSA, realizaron actividades productivas en conjunto con laborantes de la empresa como censos de cargas en varios lugares como ENICALSA, Cruz Roja León, ESESA, instalación residencial de 8 paneles solares conectados a red para disminuir el consumo de energía eléctrica del domicilio, instalación de bomba sumergible en finca agrícola ubicada en la comunidad la Ceiba, León. Consideran valiosos los aprendizajes sobre instalaciones fotovoltaica conectadas a la red como con banco de baterías, manejo con mayor facilidad de las herramientas de trabajo, motivación y entusiasmo al momento de realizar trabajos, trabajar en equipo con eficacia y rapidez y relación con clientes mediante aclaraciones técnicas.

\section{CONCLUSIÒN}

La formación de ingenieros sigue siendo clave en el siglo XXI por sus contribuciones al desarrollo de la sociedad a través de la aplicación de la ciencia. El proceso de aprendizaje en la ingeniería renovable debe ser holístico, basado en la estrategia de los saberes estratégicos como el aprender a aprender y aprender hacer sin perder la perspectiva de desarrollo sostenible inherente a la carrera.

Las prácticas de especialización en energías renovables se fundamentan en el modelo educativo UNAN Managua, en las relaciones que tiene la universidad con empresas energéticas del estado y privadas que abren sus puertas al desarrollo profesional de los estudiantes en aspectos técnicos, relaciones laborales y valores humanos. 
La integración de los estudiantes a labores productivas es un factor que incide positivamente en la sinergia de las partes: propietarios, empleados y practicante. El estudiante se motiva, estudia, aprende, se especializa y le encuentra un sentido real a su carrera. El propietario crea un valor agregado a su empresa y los empleados generosamente entrenan a las nuevas generaciones.

\section{REFERENCIAS}

Betanco M, J. A., \& Lanuza S, E. M. (2016). Programa de Asignatura Prácticas de Especialización. programa de asignatura, UNAN Managua FAREM Estelí, Ciencia Tecnología y Salud, Estelí.

Bodas Gonzáles, E. (22 de Julio de 2019). Prácticas curriculares y extracurriculares: puntos en común y diferencias. Prácticum y Prácticas Profesionales.[Blog]. Recuperado el 1 de Septiembre de 2019, de https://gidpip.hypotheses.org/3312

Capote León, G., Rizo Rabelo, N., \& Bravo López , G. (abril de 2016). La formación de ingenieros en la actualidad. Una explicación. Universidad y Sociedad, 21-28. Recuperado el 2019 de Septiembre de 01, de https://rus.ucf.edu.cu/index.php/rus

Castellanos Álvarez, Lara Díaz, L., \& Lapido Rodríguez, M. (2004). Diseño conceptual para la formación holística del ingeniero. II Taller Internacional de Docencia Universitaria. Universidad. Cienfuegos.

Castillo, J. A. (2019). Primer Informe de visita a planta Momotombo. Informe de práctica, Dirección de estudios geotérmicos, Managua.

Recuero López, M. (2002). Formación de ingeniros en España. REVISTA FACULTAD DE INGENIERÍA, U.T.A. , 10, 45-57. Recuperado el 1 de Septiembre de 2019

Rizo, N. (2007). Estrategia Didáctica de Educación en Ciencia, Tecnología y Sociedad en la Carrera de Ingeniería Informática. Tesis doctoral, Universidad de Cienfuegos, Cienfuegos. Recuperado el 2019 de septiembre de 1

Rodriguez P, M. (2016). Políticas y estrategias para la vinculación Universidad- EmpresaEstado aplicables en Nicaragua. Tesis doctoral, UNAN Managua, División de Estudios para Graduados. Doctorado en Ciencias Sociales, Managua. Recuperado el 1 de Septiembre de 2019, de http://repositorio. unan.edu.ni/3943/1/17522.pdf

UNAN Managua. (2011). Modelo Educativo, Normativa y Metodología para la Planificación Curricular. Modelo Educativo, UNAN Managua, Vicerectoria académica, Managua. Recuperado el 2019 de septiembre de 1 


\section{ANEXOS}

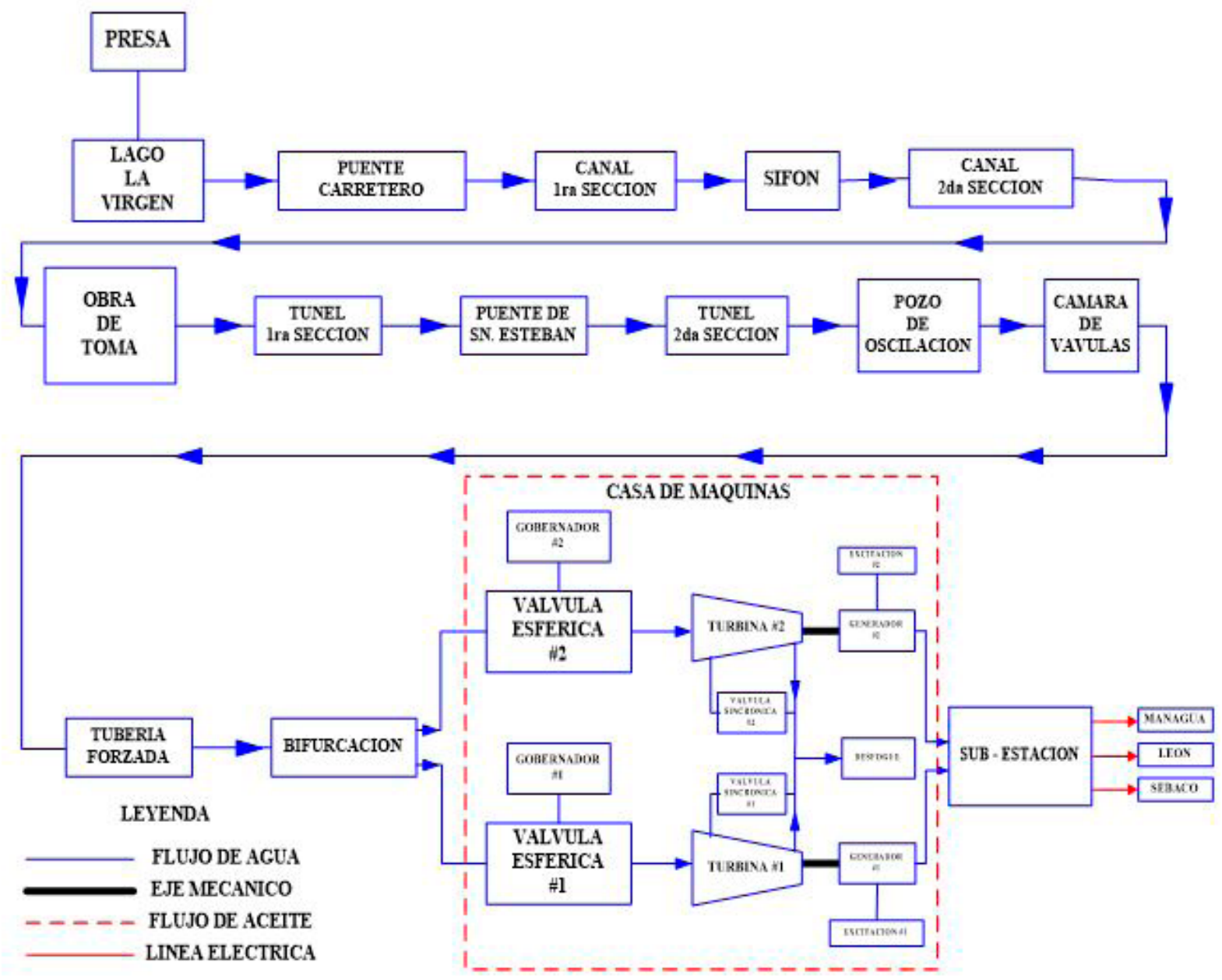

Figura 1. Esquema planta hidroeléctrica Carlos Fonseca Amador. Fuente informe de práctica Br Keydell Pastrana.

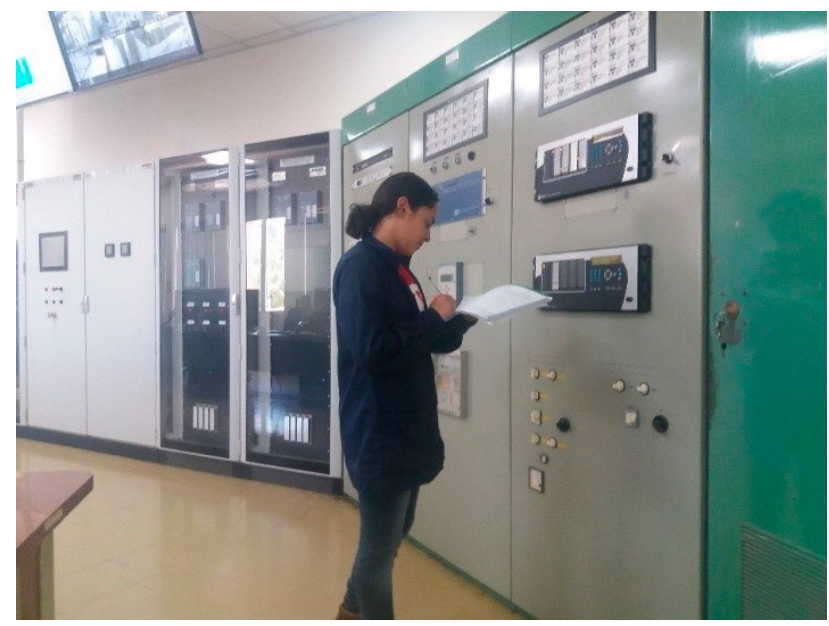

Figura 2. Control y monitoreo de elementos de planta hidroeléctrica Carlos Fonseca Amador. Fuente Br. Keydell Pastrana.

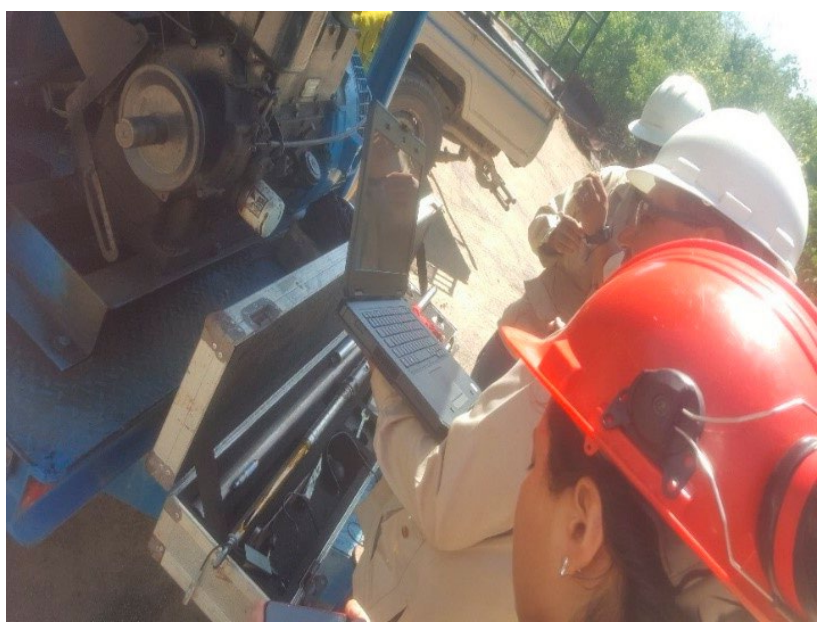

Figura 3. Preparación de registro de presión al pozo 36 de planta geotérmica Momotombo. Fuente Br. Jeffry Castillo. 


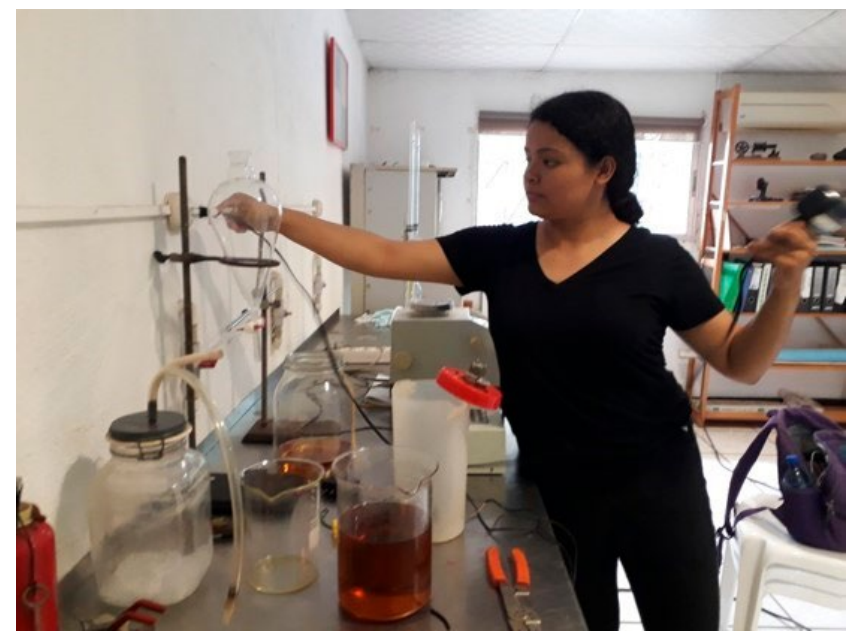

Figura 4. Pruebas de producción de biodiesel. CIDTEA, La Salle. Fuente Br. Priscila Castro.

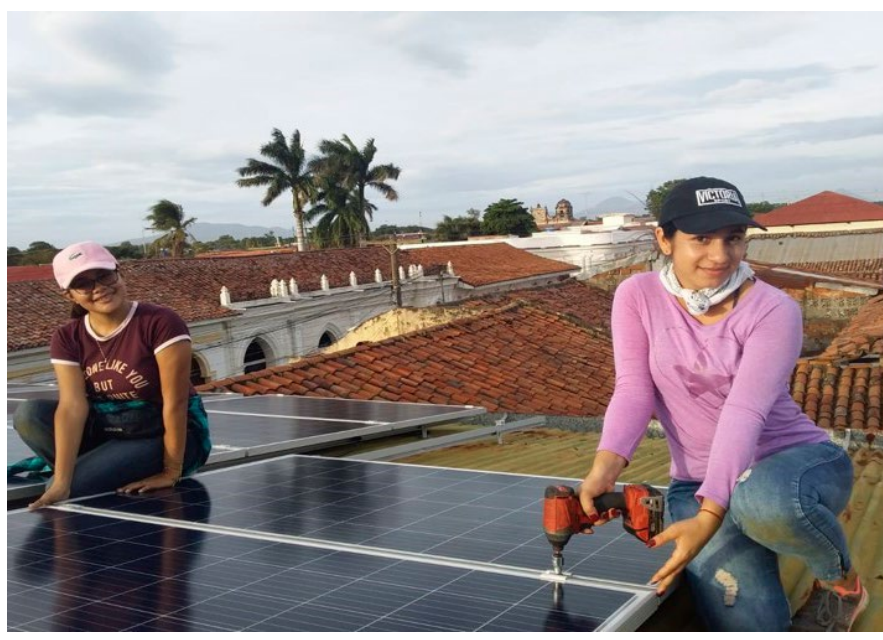

Figura 5. Instalación de sistema fotovoltaico. Fuente $\mathrm{Br}$. Rosa Melania Araúz y Br. Seyling Siles.

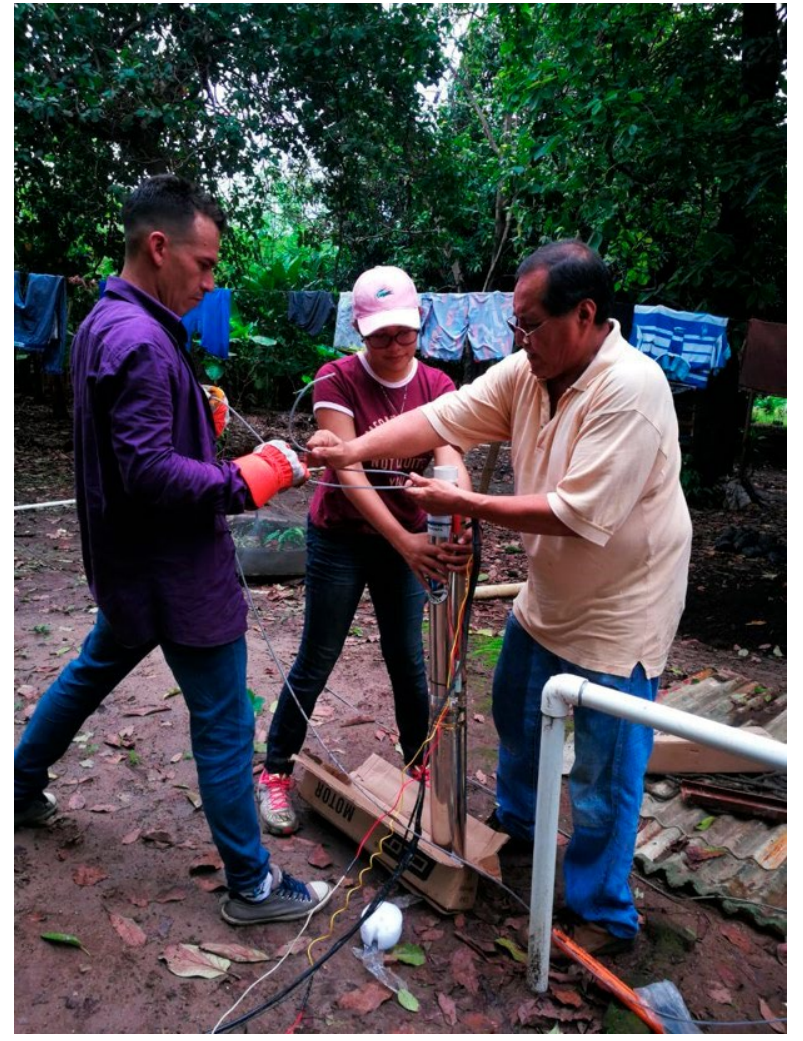

Figura 6. Mantenimiento a bombas. ECOENERGIA. Fuente: Br. Wilfredo Ramírez y Br. Rosa Melania Araúz.

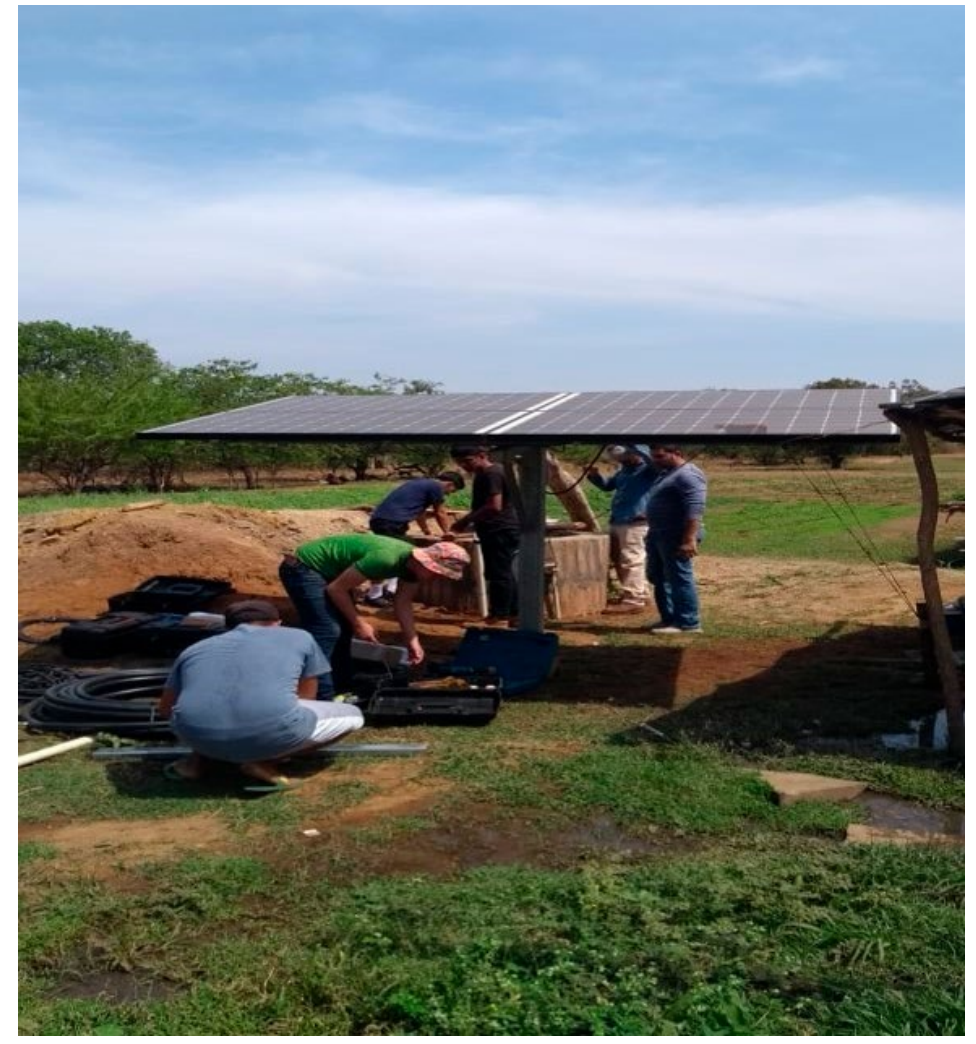

Figura 7. Instalación sistema de bombeo solar fotovoltaico. ENICALSA. Fuente: Br. Víctor Ruiz. 